TITLE:

\title{
New method of stopping power measurement for fast particles in metals and liquids
}

$\operatorname{AUTHOR}(S)$ :

Itoh, A.; Kaneda, M.; Shimizu, M.; Hayakawa, T.; Iriki, T.; Tsuchida, $\mathrm{H}$.

\section{CITATION:}

Itoh, A....[et al]. New method of stopping power measurement for fast particles in metals and liquids. Vacuum 2010, 84(8): 999-1001

\section{ISSUE DATE:}

2010-03-24

URL:

http://hdl.handle.net/2433/139419

\section{RIGHT:}

(C) 2009 Elsevier Ltd; This is not the published version. Please cite only the published version.; この論文は出版社版でありません。引用の際に は出版社版をご確認ご利用ください。 


\title{
ISI 10 \\ New Method of Stopping Power Measurement for Fast Particles in Metals and Liquids
}

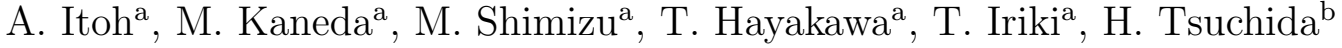 \\ ${ }^{a}$ Department of Nuclear Engineering, Kyoto University, Kyoto 606-8501, Japan \\ ${ }^{b}$ Quantum Science and Engineering Center, Kyoto University, Kyoto 606-8501, Japan
}

\begin{abstract}
A new method is developed of stopping power measurement for fast incident particles penetrating through solid and liquid targets. In this method, thin cylindrical targets are employed and it enables us to obtain the stopping powers in a rather wide range of projectile energies according to a certain incident energy. In this report, we demonstrate our recent experimental results for a metal wire of $\mathrm{Al}$ and liquid water and ethanol obtained using a molecular jet technique. We also present here the results of secondary ion emission from liquid targets measured by this method.
\end{abstract}

Key words: stopping power, liquid materials, secondary emission

\section{Introduction}

The stopping power or the stopping force $d E / d x$ of matter for energetic charged particles is the longtime subject of great importance both in fundamental and applied sciences relevant to penetration phenomena of radiations. To date, a number of research works including excellent as well as introductory review articles have been published as described in a recent textbook by Sigmund [1]. As the experimental data of stopping power and range are practically important in various application fields in materials science, biology and radiotherapy, published data covering both experiments and theories are compiled to data bases which are available in several conventional computer codes $[1,2]$. The basic concept of the stopping power at

Email address: itoh@nucleng.kyoto-u.ac.jp (A. Itoh) 
high velocities is well understood within the framework of the first Born approximation or the Bethe-Bloch theory and it provides the benchmark data in this research field. Experimentally, solids and film-like targets have been investigated in most measurements so far and less effort has been devoted particularly to liquid materials. The stopping power data and other relevant inelastic cross sections stored in available codes for compound materials like liquids are mostly those calculated by adopting the additive summation over constituent elements. However, computer simulations of track structure of charged particles in matter or dosimetric calculations in particle cancer therapy should require more reliable data of high accuracy. Hence, investigations using more realistic target materials is undoubtedly essential, in particular, for liquids like water which is the dominant component in biological cells of soft tissues. Furthermore, experimental data obtained in such a way can provide direct information to achieve better understanding of, if any, phase-difference between solid and liquid materials; e.g., ice and liquid water.

The scarcity of liquid data in atomic collision experiments is mainly due to high vapor pressure of liquids, inhibiting a good vacuum condition that is indispensable for conducting routine measurements of, for instance, stopping power and secondary particles by avoiding damages to particle-detectors such as semiconductor detectors or channel electron multipliers.

On the other hand, such a liquid-in-vacuum technique is well established in laser experiments [3], where a liquid molecular jet target is used. This technique is expected to serve as a useful tool also in atomic collision experiments of stopping power, emission of secondary particles of electrons, positive and negative ions including free radicals.

Upon this motivation, we developed recently the same method in our laboratory dedicated to the study of collision interactions with liquid phase targets in vacuum $[4,5]$. Through our experience of using this technique, we found that thin metallic wires may also be used efficiently in stopping power measurements of metal elements. This is due to a fact that the data can be obtained for a rather wide energy range of the projectile particle, though the incident energy from the accelerator remains the same. In this paper, typical advantages of this method is described along with our recent experimental results. 


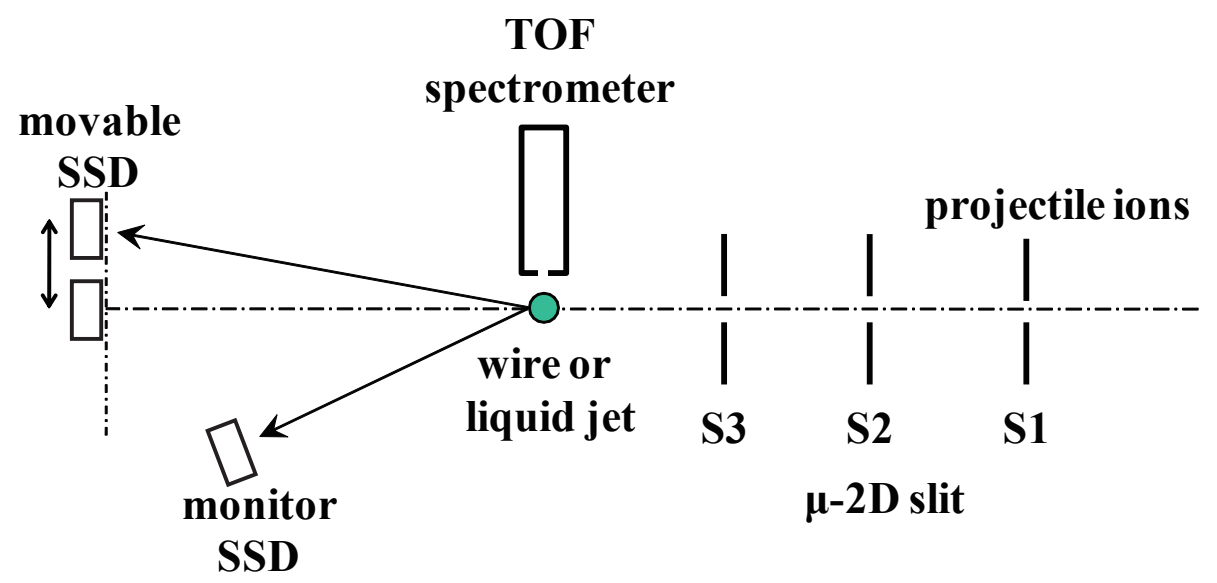

Figure 1: Experimental setup

\section{Experimental}

The experiments have been carried out at the QSEC laboratory with a Van de Graff accelerator delivering a proton beam with $\mathrm{MeV}$ energies. Figure 1 shows a schematic drawing of our experimental setup dedicated for thin wire-like targets and liquid jet targets. An incident beam from an accelerator was carefully collimated with three XY-slits adjustable with a precision of one micrometer. The beam was then incident on a wire-like target and the outgoing energy was measured with two semiconductor detectors (SSD). One of these detectors was placed at a fixed scattering angle (55 mrad) and was used as a monitor of the incident beam intensity. The second SSD was movable perpendicularly with respect to the incident beam axis and was used as an energy detector at various scattering angles. As shown in the figure, the collision chamber was also equipped with a time-of-flight (TOF) spectrometer to measure secondary ions from the target. In this case, the incident beam was chopped to a few ns in width with a repetition rate of 10 $\mathrm{kHz}$ to achieve TOF coincidence measurements. As the maximum incident energy of projectile ions available from our accelerator is limited to $2.5 \mathrm{MeV}$ at most, the thickness of target materials was thinner than several tens of micron at most, being narrower than the range of the incident beam. In this experiment, we used an $\mathrm{Al}$ wire target of $26 \mu \mathrm{m}$ in diameter. As for liquid targets of water and ethanol, we used a liquid molecular jet technique. The details of this method is described in our previous papers $[4,5,7]$. 


\section{Results and Discussion}

In comparison with other standard methods of stopping power measurements adopting film-like planar targets, the present method has the following distinctive advantage. Namely, stopping power data are possibly obtained over a rather wide range of projectile energies by using an incident beam of one fixed energy. This is due to the fact that a wire-like target has a circular cross section so that the penetration length of incident ions inside the target is not constant but varies from zero (surface) to the maximum (central) . Note here that the width of the incident beam is usually taken wider than a diameter of the wire-like target. Consequently, the outgoing energy of the beam measured by the SSD detector exhibits a continuous spectrum rather than a monoenergetic Gaussian profile observed for planner thin targets.

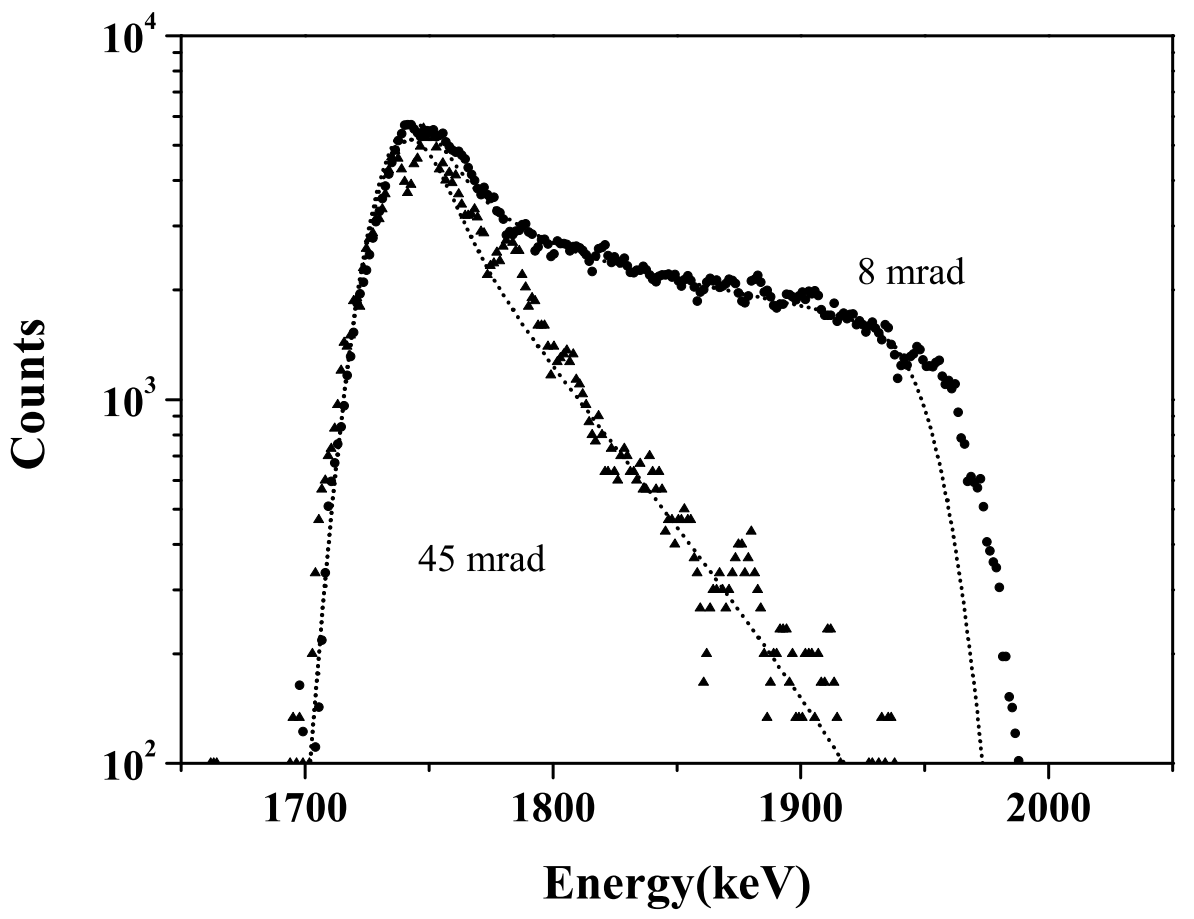

Figure 2: Energy spectra of $2.0 \mathrm{MeV}$ protons after passing through liquid ethanol scattered to angles of 8 and $45 \mathrm{mrad}$.

Actually, such continuous energy spectra are clearly demonstrated in Figs. 2 and 3 obtained for a liquid target of ethanol [5] and an Al wire, respectively. 


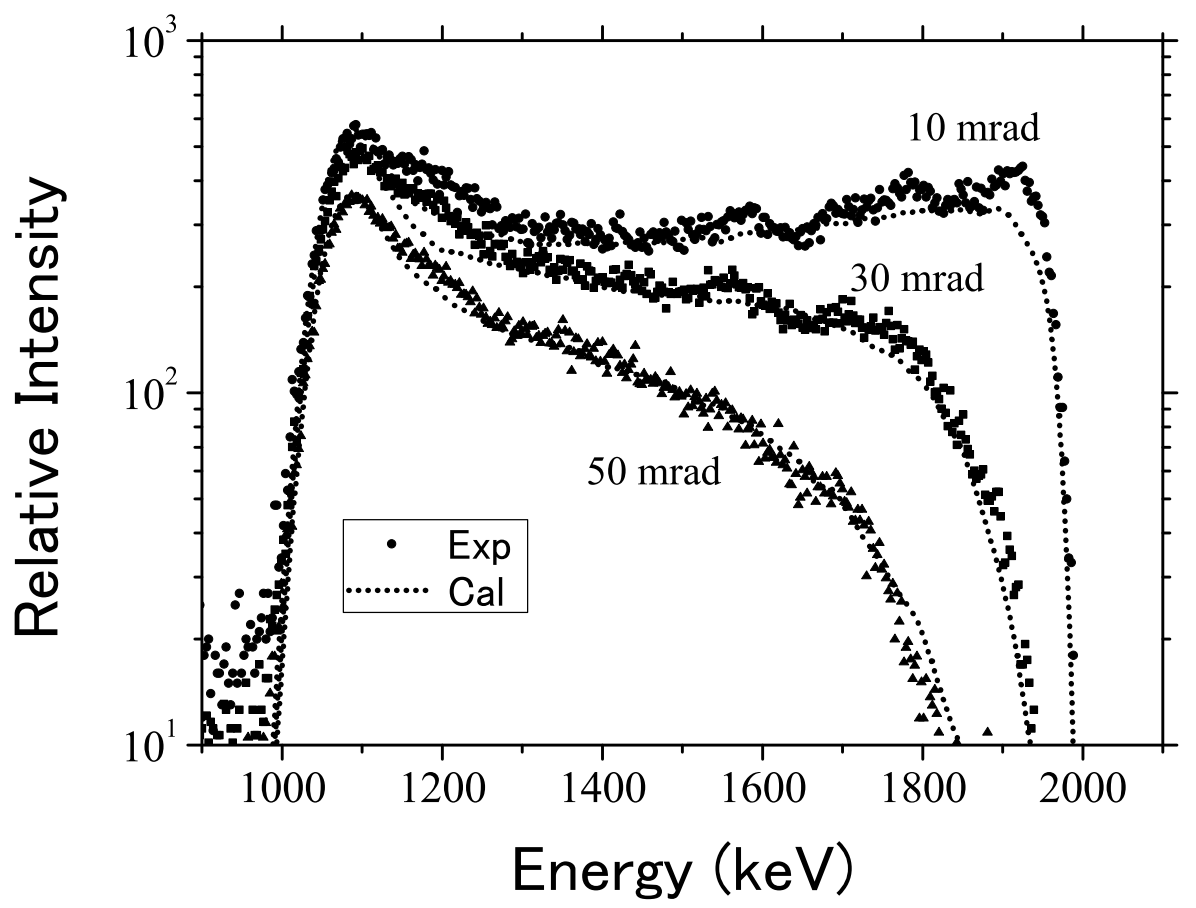

Figure 3: Energy spectra of $2.0 \mathrm{MeV}$ protons after passing through a $\mathrm{Al}$ wire of $26 \mu \mathrm{m}$ in diameter measured at scattering angles 10,30 and 50mrad 
Energy spectra in Fig. 3 are measured at scattering angles of 8 and $45 \mathrm{mrad}$, and those in Fig. 3 are 10, 30 and $50 \mathrm{mrad}$. In all the cases, the spectra are broadly spread peaking at a position of maximum energy loss. The overall shape profile may be interpreted simply by a geometrical consideration of ion trajectories inside the circular target [4].

It is noted that the maximum energy loss corresponds to those ions which passed through the center of the target. Also interesting to note is the fact that this maximum energy loss seems nearly independent of the scattering angles investigated here. Pioneering experimental results of angle-dependent energy loss of $\mathrm{MeV}$ protons show that the difference appears at scattering angles larger than 1 degrees [6], which are comparable angles to our case. The present results indicate that the angle-dependency of energy loss may be smeared out due to numerous multiple scatterings of small angles. Actually, the number of collisions is estimated to be on the order of $10^{4}$ in our experiments. Apart from these boundaries, a series of experimental spectra allow us to treat the energy loss of fast particles scattered to several tens of mrad in the same way as those ions emerging to the zero-degree direction. On the other hand, however, the energy spectra are surprisingly different from each other at these small scattering angles. This is our key idea, stressing that the energy spectra at small scattering angles are highly promising to determine the stopping power accurately. The procedure is as described in $[5,8]$.

Since an experimental spectrum is a result of superposition of various energy spectra of individual particles experiencing different scattering processes, the spectrum may be reproduced numerically by calculations of energy losses in individual small-angle scatterings with use of appropriate stopping power data and interatomic potentials. For this Monte Carlo simulation, we used either the Moliere theory of multiple scattering or the GEANT4 code and two types of interatomic potentials (ZBL and Bohr) are used. The stopping power data used in the calculations are taken as, $S(E)=d E / d x=\alpha S_{r e f}(E)$, where $E$ is the energy of incident particles, $S_{r e f}$ the reference data available from the SRIM code or the ICRU-49 report, and $\alpha$ is the fitting parameter. The diameter of the target is also treated as the second fitting parameter. Iterative fitting calculations are made until the experimental energy spectra at different emerging angles are reproduced. The simulation results are also plotted in Figs 2 and 3 by dotted lines, showing excellent agreement with all the experimental data. In particular, better agreement obtained for liquid ethanol in comparison with the $\mathrm{Al}$ target seems 


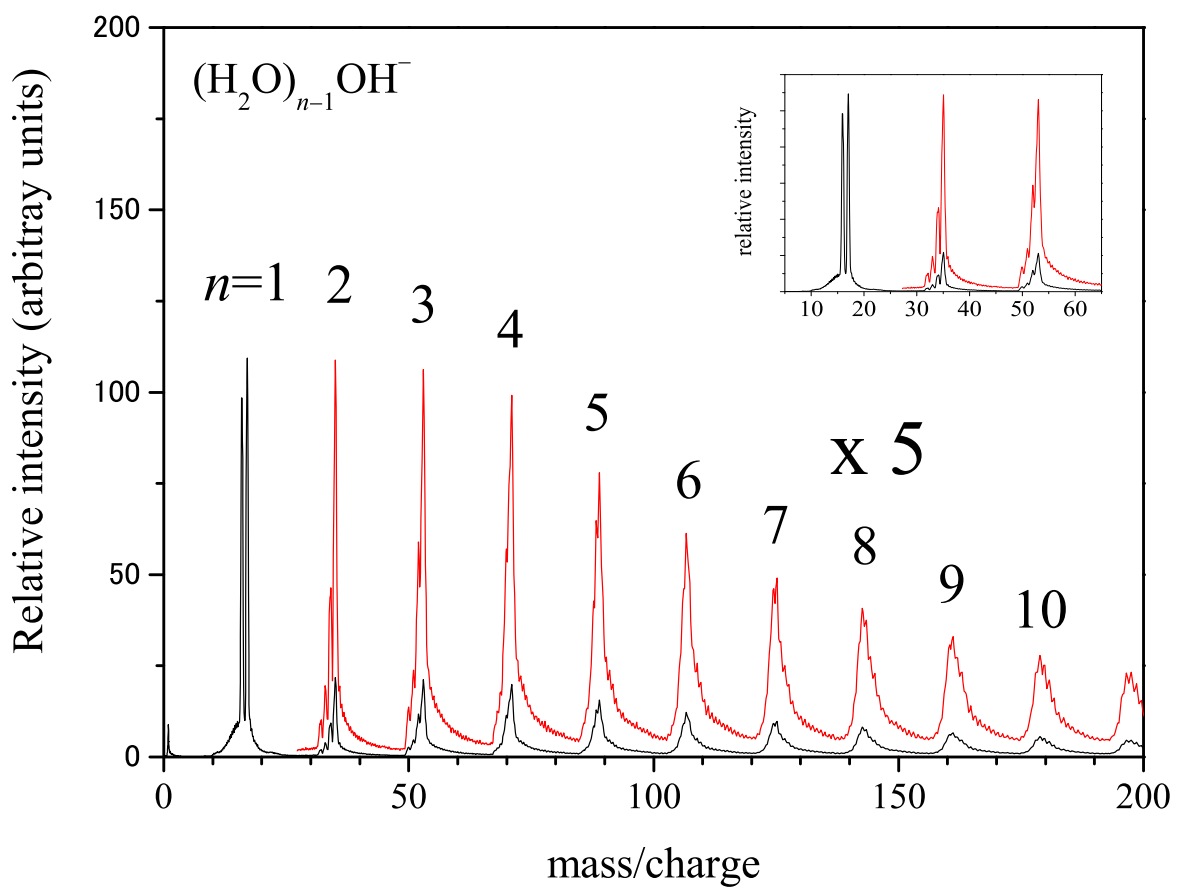

Figure 4: Negative cluster ions produced from liquid water bombarded by $2 \mathrm{MeV} \mathrm{He}{ }^{+}$ ions 
to imply that the liquid target has a more circular-shape cross section than $\mathrm{Al}$. In this way, we found that the stopping powers of ethanol for fast protons is smaler than the SRIM data by about $20 \%$ in a range from 1.5-2.0 $\mathrm{MeV}$, while those of $\mathrm{Al}$ coincide fairy well, as expected, with available data in a range from 1.8 to $2.5 \mathrm{MeV}$. It is noted that the stopping powers can be determined within uncertainties of a few percents.

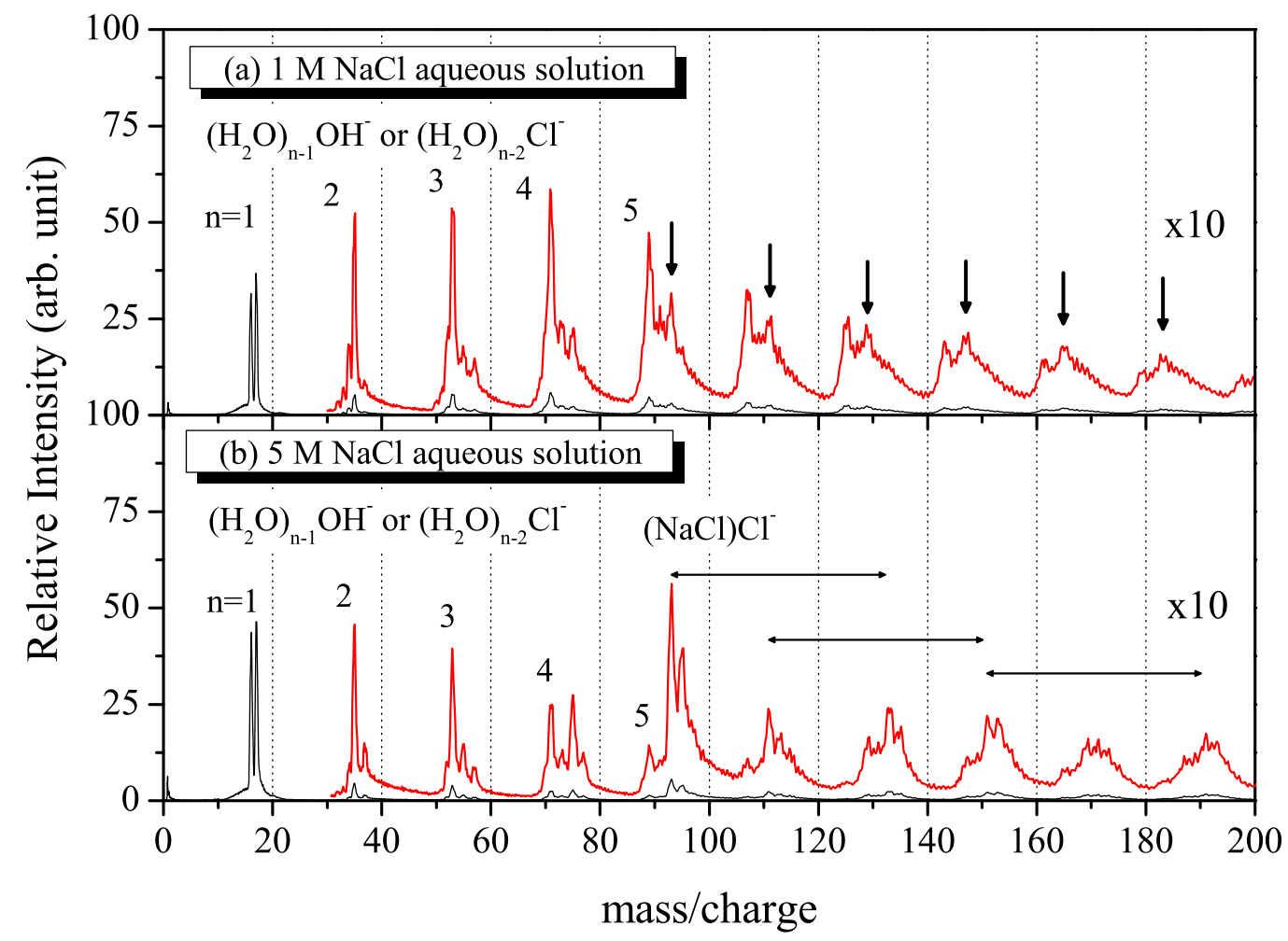

Figure 5: Negative ion spectra from $\mathrm{NaCl}$ aqueous solutions of (a) $1 \mathrm{M}$ and (b) $5 \mathrm{M}$.

As an extension of our experimental method, secondary ion emission was also investigated successfully. Figure 4 shows a TOF spectrum of secondary negative ions produced from liquid water bombarded by $2 \mathrm{MeV}$ $\mathrm{He}^{+}$ions [7]. In this kind of measurement, we used a time- flight-tube with a tiny entrance hole (1 $\mathrm{mm}$ in diameter), which is separated from the collision chamber and is differentially pumped by another turbo molecular pump. Negative ion spectrum shows a series of cluster ions of $\left(\mathrm{H}_{2} \mathrm{O}\right)_{n} \mathrm{OH}^{-}$, 
$\left(\mathrm{H}_{2} \mathrm{O}\right)_{n} \mathrm{O}^{-},\left(\mathrm{H}_{2} \mathrm{O}\right)_{n-1} \mathrm{HO}_{2}^{-}$and $\left(\mathrm{H}_{2} \mathrm{O}\right)_{n-1} \mathrm{O}_{2}^{-}$(see an inset).

Another example of the cluster ion production from liquid is demonstrated in Fig. 5 for the targets of $\mathrm{NaCl}$ aqueous solutions with different concentration [7]. One can see the spectral profile change strongly as the concentration of $\mathrm{NaCl}$ increases. In the upper figure (1.0M), negative ions observed strongly at mass/charge $18 \mathrm{n}+17$ are attributable to $\left(\mathrm{H}_{2} \mathrm{O}\right) \mathrm{OH}^{-}$and $\left(\mathrm{H}_{2} \mathrm{O}\right) \mathrm{Cl}^{-}$. Furthermore, the intensity ratios of Na-contained ions $(18 \mathrm{n}+21)$ to Na-noncontained ions $(18 \mathrm{n}+17)$ becomes larger with increasing cluster size $n$. As for the lower figure $(5.0 \mathrm{M})$, on the contrary, these ions decrease rapidly with increasing $n$, and $(\mathrm{NaCl}) \mathrm{Cl}^{-}$is the most intensive ions among large cluster ions. Distinctive peaks located with mass intervals of 58 can be attributed to attachment of $(\mathrm{NaCl})_{n}$ ions.

In summary, we demonstrated a new experimental method to obtain various atomic collision data relevant to stopping power and secondary emission from wire-like metal targets including also volatile liquids like water. The present method is undoubtedly a promising technique to achieve a kind of breakthrough in atomic collision physics relevant to liquid materials.

This work was supported by a Grant-in-Aid for Scientific Research (B) 19360427 from the Japan Society for the Promotion of Science.

\section{References}

[1] P. Sigmund, Particle penetration and radiation effects, Vol. bf 151 in Solid-State Sciences, Springer, ISSN 0171-1873 (2006)

[2] H. Paul, Nucl. Instr. and Meth. B,bf 247 (2006) 166

[3] M. Faubel, S. Schlemmer, J.P. Toennies, Z. Phys. D 10 (1988) 269.

[4] A. Itoh, M. Kaneda, S. Satoh, K. Ishii, and H. Tsuchida, Nucl. Instr. and Meth. B 245 (2006)76

[5] M. Kaneda, S. Sato, M. Shimizu, Z. He, K. Ishii, H. Tsuchida, and A. Itoh, Nucl. Instr. and Meth. B 256 (2007) 97.

[6] R. Ishiwari, N. Shiomi and N. Sakamoto, Phys. Rev. A 25 (1982) 2524.

[7] M. Kaneda, M.Shimizu, T. Hayakawa, A. Nishimura, Y. Iriki, H. Tsuchida, et. al., Nucl. Instr. and Meth. B 267 (2009) 908 
[8] M. Shimizu, M. Kaneda, T. Hayakawa, T. Tsuchida and A. Itoh, Nucl. Instr. and Meth. B 267 (2009) 2667 